\title{
Optimal regular reflection of shock and blast waves
}

\author{
Mihail Chernyshov ${ }^{1, *}$, and Alexandr Tyapko ${ }^{2}$ \\ ${ }^{1}$ Baltic State Technical University "VOENMEH", St. Petersburg, Russia \\ ${ }^{2}$ Peter the Great St. Petersburg Polytechnic University, St. Petersburg, Russia
}

\begin{abstract}
The regular reflection of an oblique steady shock in supersonic gas flow is considered. The static pressure extremum conditions after the point of reflection of the shock with fixed strength depending on oncoming flow Mach number are determined analytically. The obtained results are applied to solution of the mechanically equivalent problem of the reflection of a propagating shock from an inclined surface. Non-monotonic variation of the mechanical loads on the obstacle with respect to its inclination angle is shown; the obstacle slope angles that correspond to pressure minima downwards of the unsteady shock reflection point are determined analytically.
\end{abstract}

\section{Introduction}

The regular reflection of steady and unsteady shocks from a rigid surface or a flow symmetry plane is well-studied phenomenon. If the ratio $\gamma$ of the gas specific heats in the steady supersonic flow is known and fixed, then the reflected shock parameters depend solely on the oncoming flow Mach number $M$ (Fig. 1a) and one additional parameter of the incident shock (for example, its strength $J_{1}$ or its amplitude $\Delta p_{1}$ ). Here the strength $J_{1}$ of the incident shock is the static pressure ratio across it, and the amplitude $\Delta p_{1}$ is the difference between pressures while crossing the shock (the shock overpressure which is especially important in problems of blast reflection $[1,2]$ and corresponding structural survivability [35]).

The reflected shock strength $J_{2}\left(M, J_{1}\right)$ and its amplitude (overpressure) $\Delta p_{2}\left(M, \Delta p_{1}\right)$ are monotonic functions of the strength $J_{1}$ (or the amplitude $\Delta p_{1}$ ) of the incident perturbation. It is proven analytically in the present study that the reflected shock parameters are non-monotonic functions of the flow Mach number $M$, and the pressure extreme conditions downstream of the reflected shocks are determined analytically.

\footnotetext{
*Corresponding author: $\underline{\text { mvcher@mail.ru }}$
} 

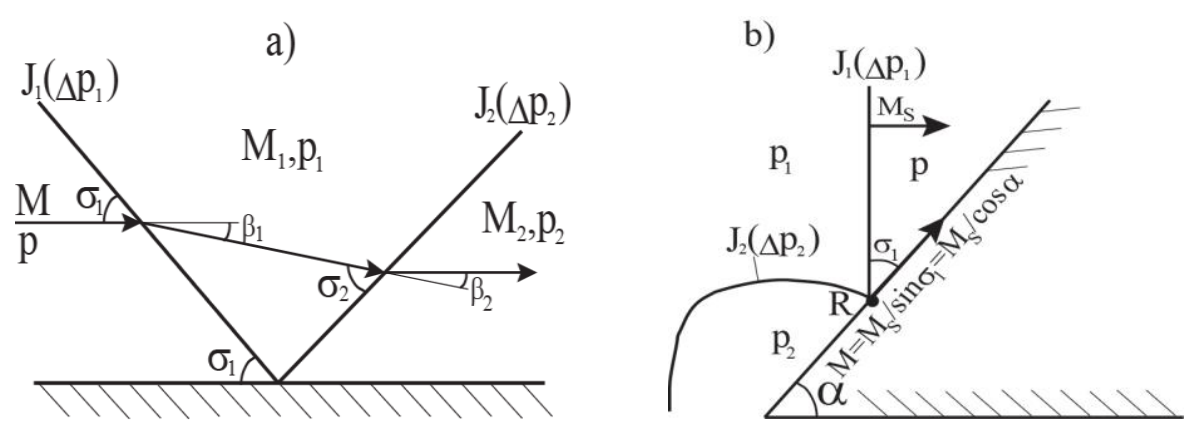

Fig. 1. Regular reflection of oblique shock $(a)$ and the equivalent unsteady shock reflection from the inclined obstacle $(b)$

The engineering value of the obtained analytical solutions is based on the equivalent problem of oblique reflection of propagating shock (blast) waves, see Fig. 1b. The pressure downstream of the reflection point of an oblique steady shock at an incidence angle $\sigma_{1}$ (Fig. 1a) is equal to the pressure after the reflection point of a moving shock having the same strength (amplitude) from a wedge inclination angle of $\alpha=\pi / 2-\sigma_{1}$ (Fig. 1b) relative to the direction of shock propagation. The variation of flow Mach number $M$ in the steady problem corresponds to variation in the wedge angle $\alpha$ in the problem of unsteady (quasisteady) reflection of propagating shock of the same strength. Thus, the non-monotonic dependence of the mechanical loads behind reflected unsteady shock from an inclined surface appears from the non-monotonic pressure variation downstream the point of steady reflection.

\section{Model and methods}

\subsection{Governing equations for the strength of the reflected shock}

The oncoming flow parameters (Mach number $M$, pressure $p$ and the gas specific heat ratio, $\gamma)$ and the shock slope angle $\sigma_{1}$ determined its strength [6-8]: $J_{1}=p_{1} / p=(1+\varepsilon) M^{2} \sin ^{2} \sigma_{1}-\varepsilon$, its amplitude: $\Delta p_{1}=p_{1}-p=p \cdot\left(J_{1}-1\right)$, and the flow deflection angle $\beta_{1}$ behind the incident shock, as well as Mach numbers after the shocks. The condition of regular reflection,

$$
\beta_{1}+\beta_{2}=0
$$

leads to the equation concerning the strength $J_{2}=p_{2} / p_{1}$ of the reflected shock:

$$
\begin{gathered}
\sum_{n=0}^{3} C_{n} J_{2}^{n}=0 \\
C_{3}=\left[\left(1+\varepsilon J_{1}\right) J_{1} M\right]^{2} \\
C_{2}=-J_{1}\left(1+\varepsilon J_{1}\right) \cdot\left[(1+\varepsilon)\left(J_{1}+\varepsilon\right) M^{4}+\left(\varepsilon(2-\varepsilon) J_{1}^{2}-\varepsilon(1-2 \varepsilon) J_{1}+2\left(1-\varepsilon^{2}\right)\right) M^{2}-(1-\varepsilon)\left(J_{1}+\varepsilon\right)\left(J_{1}-1\right)^{2}\right] \\
C_{1}=(1+\varepsilon)\left(J_{1}+\varepsilon\right)\left(1+(1+2 \varepsilon) J_{1}^{2}\right) M^{4}-x_{1} M^{2}+(1-\varepsilon)\left(J_{1}+\varepsilon\right)\left(J_{1}+1\right)\left(J_{1}-1\right)^{3}
\end{gathered}
$$




$$
\begin{gathered}
C_{0}=-(1+\varepsilon)\left(J_{1}+\varepsilon\right)\left((1+2 \varepsilon) J_{1}-\varepsilon\right) M^{4}+x_{0} M^{2}-(1-\varepsilon)\left(J_{1}+\varepsilon\right)^{2}\left(J_{1}-1\right)^{2}, \\
x_{1}=2(1+\varepsilon(1-\varepsilon)) J_{1}^{4}-2\left(1-2 \varepsilon^{2}\right) J_{1}^{3}-\varepsilon\left(4-\varepsilon-2 \varepsilon^{2}\right) J_{1}^{2}+2 \varepsilon(1-\varepsilon) J_{1}-\left(1-2 \varepsilon^{2}\right), \\
x_{0}=2\left(1+\varepsilon-\varepsilon^{2}\right) J_{1}^{3}-\left(2-6 \varepsilon^{2}+\varepsilon^{3}\right) J_{1}^{2}-2 \varepsilon(1-\varepsilon)(1+2 \varepsilon) J_{1}+\varepsilon\left(1-2 \varepsilon^{2}\right) .
\end{gathered}
$$

\subsection{Limiting values for the strength of the incident shock}

Satisfactory solution of (1) exists, if the incident shock strength obeys the condition,

$$
1 \leq J_{1} \leq J_{d}(M) \text {. }
$$

Here $J_{d}(M)$ is the shock strength that corresponds to the so-called detachment criterion $[1,9]$ of the reflection transition. The value of $J_{d}(M)$ corresponds to the curve 1 in Fig. 2a. Here and further on all computed examples are obtained at $\gamma=1.4$.

The von Neumann criterion of the mechanical equilibrium is used also at moderate and large Mach numbers $\left(M>M_{a}, M_{a}=\sqrt{(2-\varepsilon) /(1-\varepsilon)}=1.483\right)$. Corresponding strength $J_{N}(M)$ is presented by curve 2 in Fig. 2 a.

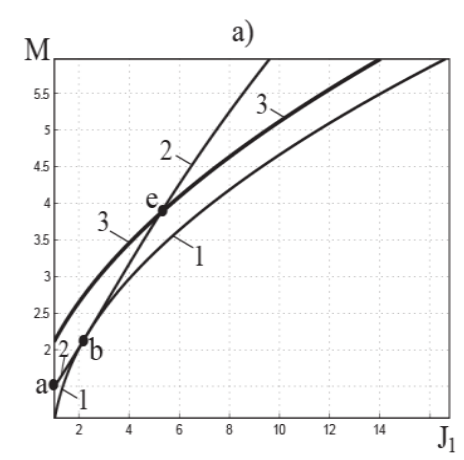

b)

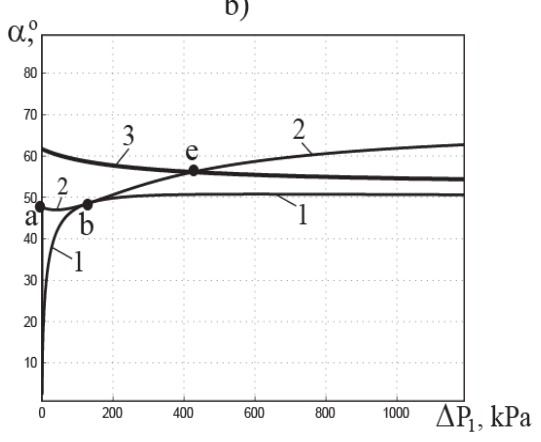

c)

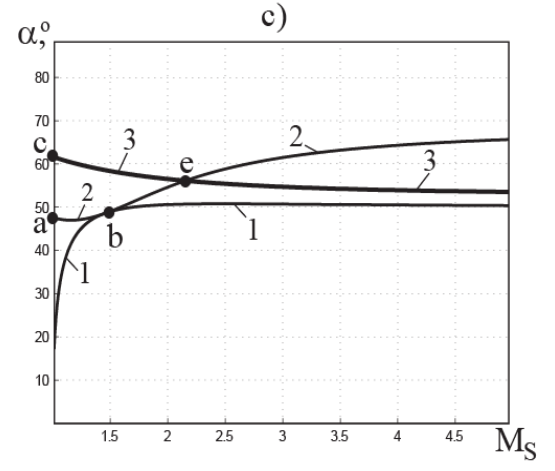

Fig. 2. Limting conditions of strength and amplitude extreme for regularly reflected shock

Solutions for both regular and Mach reflection co-exist in the dual solution domain situated between curves 1 and 2 . Numerous experiments $[1,10]$ shows that the criterion of the mechanical equilibrium is preferable for steady flow analysis, especially in the part of the 
dual solution domain situated above point $b$, the contact point between curves 1 and 2. Flow Mach number $M_{b}=2.202$ corresponds to the following equation [11, 12]:

$$
\begin{gathered}
\sum_{n=0}^{4} F_{n} M_{b}^{2 n}=0, F_{4}=(1-\varepsilon)\left(2-4 \varepsilon+2 \varepsilon^{3}-\varepsilon^{4}\right), \\
F_{3}=-10+20 \varepsilon-10 \varepsilon^{2}-10 \varepsilon^{3}+12 \varepsilon^{4}-4 \varepsilon^{5}, \\
F_{2}=12-24 \varepsilon+10 \varepsilon^{2}+16 \varepsilon^{3}-18 \varepsilon^{4}+6 \varepsilon^{5}, F_{1}=-2(1+\varepsilon)\left(3-4 \varepsilon+2 \varepsilon^{2}\right)(1-\varepsilon)^{2}, \\
F_{0}=(1+\varepsilon)(1-\varepsilon)^{4} .
\end{gathered}
$$

Since solutions derived for steady flows will be applied later to unsteady shock reflections, we consider now the entire range (2) of incident shock parameters.

\subsection{The equivalent unsteady flow with regular reflection of the propagating shock}

After the inversion of motion, the reflection of the moving shock of the same strength,

$$
J_{1}=p_{1} / p=(1+\varepsilon) M^{2} \sin ^{2} \sigma_{1}-\varepsilon=(1+\varepsilon) M^{2} \cos ^{2} \alpha-\varepsilon,
$$

that propagates in a stagnant gas media and reflects from a wedge having deflection angle $\alpha=\pi / 2-\sigma_{1}$ (Fig. 1b) is mechanically equivalent to the regular steady reflection shown in Fig. 1a [13]. Reflection point $R$ (Fig. 1b) moves along the wedge surface with the same Mach number $M$ as the oncoming flow in a similar steady problem. Then the normal velocity of the incident shock corresponds to Mach number $M_{S}=M \sin \sigma_{1}=M \cos \alpha=\sqrt{\left(J_{1}+\varepsilon\right) /(1+\varepsilon)}$, so that the incident shock strength is $J_{1}=(1+\varepsilon) M_{S}^{2}-\varepsilon$. The strength $J_{2}$ of the reflected shock and the static pressure behind it are the same as in the equivalent case of a steady reflection.

The amplitude of the incident shock is:

$$
\Delta p_{1}=p_{1}-p=p \cdot\left(J_{1}-1\right),
$$

the amplitude of the reflected wave is equal to $\Delta p_{2}=p_{2}-p_{1}=p J_{1} \cdot\left(J_{2}-1\right)$, and the overpressure after the reflection point is $\Delta p_{\Sigma}=p_{2}-p=p \cdot\left(J_{1} J_{2}-1\right)$.

It is therefore apparent that the reflected wave amplitude $\Delta p_{2}$ and the overpressure $\Delta p_{\Sigma}$ , at fixed values of $p$ and $J_{1}$, depend linearly on the strength $J_{2}$. Therefore, $\Delta p_{2}$ and $\Delta p_{\Sigma}$ depend on the wedge angle $\alpha$ in the unsteady case and on the Mach number $M$ in an equivalent steady case qualitatively as $J_{2}$ depends on those factors. Non-monotonic variation of $J_{2}(M)$ at a regular steady reflection means that the overpressure $\Delta p_{\Sigma}$ after the point of reflection of the propagating shock also depends on the reflecting surface angle non-monotonically. The described fact seems not evident and needs proof.

The pressure ratio across the reflected wave, $K_{2}$ characterizes its amplitude relative to the incident shock:

$$
K_{2}=\Delta p_{2} / \Delta p_{1}=\left(J_{2}-1\right) /\left(J_{1}-1\right) \text {. }
$$


The pressure amplification factor $K_{\Sigma}$ characterizes the load increase on a considered obstacle:

$$
K_{\Sigma}=\Delta p_{\Sigma} / \Delta p_{1}=\left(J_{1} J_{2}-1\right) /\left(J_{1}-1\right)
$$

Both coefficients are minimal $\left(K_{2}=0, K_{\Sigma}=1\right.$ ) in the case of a sliding shock and the reflected shock disappears (degenerates to a weak disturbance, $J_{2}=1$ ). The amplitude of a normally reflected shock ( $\alpha=90^{\circ}, \sigma_{1}=0$ ) can be evaluated using the Crussard-Izmaylov formula [14]:

$$
\Delta p_{2}=\Delta p_{1} \cdot\left[1+\Delta p_{1} /\left(\varepsilon \Delta p_{1}+(1+\varepsilon) p_{0}\right)\right]
$$

with corresponding values of the factors of pressure (5) and amplification (6).

\section{Results.}

Applying the equation $\partial J_{2} / \partial M=0$ to (1) determines the conditions for extreme strength of the reflected steady shock:

$$
\begin{gathered}
\sum_{n=0}^{3} G_{n} J_{1}^{n}=0, \\
G_{3}=(1-\varepsilon)^{2}, G_{2}=-3(1-\varepsilon) \cdot\left[(1+\varepsilon) M^{2}+1-\varepsilon\right], \\
G_{1}=(1-\varepsilon) \cdot\left[(1+\varepsilon)(3+2 \varepsilon) M^{4}+2(1+\varepsilon)(1-2 \varepsilon) M^{2}+(1-\varepsilon)(1-2 \varepsilon)\right], \\
G_{0}=-\left[(1+\varepsilon) M^{4}+1-\varepsilon\right] \cdot\left[(1+\varepsilon)(1-2 \varepsilon) M^{2}-(1-\varepsilon)(1+2 \varepsilon)\right],
\end{gathered}
$$

Solution of (7), shown by curve 3 in Fig. 2 a, indicates that it really corresponds to the reflected shock strength minima and pressure minima downstream of it.

The plane $\left(\Delta p_{1}, \alpha\right)$ shown in Fig. $2 \mathrm{~b}$ is convenient for analyzing the equivalent unsteady problem. Relation (4) connects between the incident shock amplitude and its strength, relation (3) connects between the surface slope angle and the Mach number and shock strength for $\alpha=90^{\circ}-\sigma_{1}$. It was also confirmed that the shock wave propagates in undisturbed quiescent media having initial pressure of $p=100 \mathrm{kPa}$.

The regular reflection solution exists in the wedge angles range of: $\alpha \in\left[\alpha_{d}, 90^{\circ}\right)$. Angle $\alpha_{d}$ (curve 1 in Fig. 2b) corresponds to transition to irregular reflection. Curve 2 corresponds to criterion of the mechanical equilibrium. Curve 3 points to the obstacle angles that provide reflected pressure minima at any fixed amplitude of the incident shock.

Surface angles that yield minima in the pressure loads are also demonstrated in coordinates $\left(M_{S}, \alpha\right)$, Fig. 2c. The curve $2 \mathrm{c}$ corresponds to pressure minima after the point of reflection of the shock wave with some definite Mach number $M_{S}$ of its propagation.

"Optimal" surface inclination angles obey the following equation in coordinates $\left(M_{S}, \alpha\right)$ :

$$
\sum_{n=0}^{3} Z_{n} z^{n}=0
$$




$$
\begin{gathered}
z=\sin ^{2} \sigma_{1}=\cos ^{2} \alpha, Z_{3}=(1-\varepsilon)^{2}\left(M_{S}^{2}-1\right) \cdot\left[(1+\varepsilon) M_{S}^{4}-2(1+\varepsilon) M_{S}^{2}-(1-\varepsilon)\right] \\
Z_{2}=-(1-\varepsilon) M_{S}^{2} \cdot\left[3(1+\varepsilon) M_{S}^{4}-2(1+\varepsilon) M_{S}^{2}+(1-\varepsilon)\right] \\
Z_{1}=-(1-\varepsilon) \cdot\left[(3+2 \varepsilon) M_{S}^{2}+1-2 \varepsilon\right], Z_{0}=(2 \varepsilon-1) M_{S}^{6} .
\end{gathered}
$$

According to (8), at the limiting reflection case of weak shocks $\left(M_{S} \rightarrow 1\right)$, the pressure minimum corresponds to $z=(\sqrt{2-2 \varepsilon}-1) / \sqrt{2-2 \varepsilon}=0.225$, and the optimal wedge angle becomes equal to $\alpha_{c}=\arcsin (2-2 \varepsilon)^{-1 / 4}=\arcsin [(\gamma+1) / 4]^{1 / 4}=61.656^{\circ}$ (curve 3, point $c$ ). The optimal angle corresponds to the von Neumann criterion at $M_{S}=2.175$ (point $e$ of intersection of curves 2 and 3). Its value ( $\alpha_{e}=56.105^{\circ}$ ) obeys the relation

$$
z_{e}=\cos ^{2} \alpha_{e}=\frac{(1-\varepsilon)(3-\varepsilon)(1-2 \varepsilon)^{2}-\sqrt{3-20 \varepsilon+49 \varepsilon^{2}-52 \varepsilon^{3}+20 \varepsilon^{4}}}{(1-\varepsilon)(3-2 \varepsilon)\left(1-5 \varepsilon+2 \varepsilon^{2}\right)}=0.311 \text {. }
$$

At $M_{S}>2.175$, the optimal wedge inclination angle corresponds to the dual solution domain situated between curves 1 and 2 .

In the limiting case of strong wave reflection $\left(M_{S} \rightarrow \infty\right)$, the optimal obstacle angle obeys the following equation for the horizontal asymptote of curve 3 :

$$
(1-\varepsilon)\left(1-\varepsilon^{2}\right) z^{3}-3\left(1-\varepsilon^{2}\right) z^{2}+(1-\varepsilon)(3+2 \varepsilon) z+2 \varepsilon-1=0
$$

( $z=0.367, \alpha=52.715^{\circ}$ ). It lays between the horizontal asymptotes of curves 1 and 2. As seen from Figs $2 \mathrm{~b}$ and $2 \mathrm{c}$, the "optimal" surface slope angle shifts closer to the lower border of the region for regular reflection for the case of stronger shocks.

\section{Discussion}

Dependence of the reflected wave pressure and amplification coefficients $(5,6)$ on the obstacle slope angle $\alpha$ is demonstrated in Fig. 3,a-b, for various amplitudes of the incident shock (curves 1-10). Slope angle $\alpha$ changes from curve 11 that corresponds to the detachment criterion, to the value $\alpha=90^{\circ}$ that corresponds to the normal reflection as a limiting case for the regular one. The Crussard-Izmaylov relation is appropriate in the latter case. It is seen in Fig. 4a that meaningful pressure decrease is obtained behind the reflected shock.

The obtained non-monotonicity of reflected overpressure dependence on the obstacle inclination angle follows immediately from well-known relations for oblique shocks. So the theory of optimization of shock-wave systems, leads to better engineering solutions in many problems of jet and blast technologies, aviation and rocket engineering, blast protection and supersonic aerodynamics. 
a)

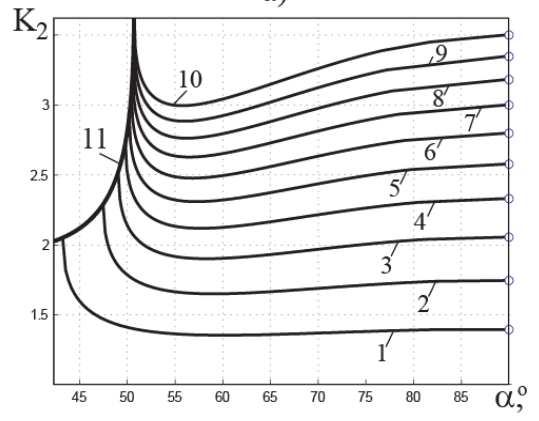

b)

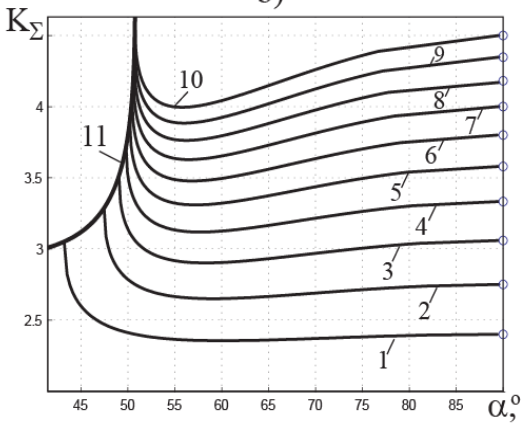

Fig. 3. Variation of the reflected pressure factor $(a)$ and pressure amplification factor $(b)$ depending on obstacle inclination angles at several fixed amplitudes of the incident shock: $50 \mathrm{kPa}$ (curves 1), $100 \mathrm{kPa}$ (2), $150 \mathrm{kPa}$ (3), $200 \mathrm{kPa}$ (4), $250 \mathrm{kPa}(5), 300 \mathrm{kPa}$ (6), $350 \mathrm{kPa}$ (7), $400 \mathrm{kPa}$ (8), $450 \mathrm{kPa}$ (9), $500 \mathrm{kPa}$ (10). Curves 11 border the domain of regular reflection existence according to the detachment criterion

\section{Conclusion.}

Flow Mach numbers and parameters of the incident shocks that achieve minima in the static pressure downstream of the reflected shocks obey the cubic algebraic equation in "shock strength - oncoming flow Mach number" variables. The analogous equation defines the obstacle slope angles that yield a minimum pressure after the reflection of the propagating shock having any amplitude. Those optimal angles exist at all theoretically possible parameters of the incident shock. The optimal shock reflection differs both from the normal one and from limiting case of shock reflection transition. The geometrical optimization of shock/blast wave interaction with obstacles and constructions allows to diminish the impulse mechanical loads significantly. The obtained theoretical results can be applied, at least, for design of blast-resistant constructions, blast protection equipment, in aviation and rocket engineering, and in supersonic aerodynamics.

This work was financially supported by the Ministry of Education and Science of the Russian Federation (agreement No. 14.577.21.0277, a unique identifier of applied scientific research RFMEFI57717X0277).

\section{References}

1. G. Ben-Dor, Shock Wave Reflection Phenomena, Springer, Berlin - Heidelberg - New York, 2007

2. M.V. Silnikov, A.I. Mikhaylin, Acta Astronautica 97, 30-37 (2014)

3. M.V. Gravit, O.V. Nedryshkin, O.T. Ogidan, Magazine of Civil Engineering 1, 38-46 (2018)

4. O. Nedryshkin, M. Gravit, K. Grabovyy, MATEC Web of Conferences 193, 03023 (2018)

5. M.V. Gravit, E.V. Golub, S.P. Antonov, Magazine of Civil Engineering 3, 86-94 (2018)

6. V.V. Selivanov, I.F. Kobylkin, S.A. Novikov Blast Technologies. Moscow: Bauman Moscow State Technical University, 648 (2008)

7. A. Chpoun, G. Ben-Dor, Shock Waves 5, 199-204 (1995) 
8. M.S. Ivanov, S.F. Gimelshein, A.E. Beylich, Hysteresis effect in stationary reflection of shock waves, Physics of Fluids, 7 (1995) 685-687. [16] H.G. Hornung, M.I. Robinson, Journal of Fluid Mechanics 123, 155-164 (1982)

9. H.G. Hornung, Annual Review of Fluid Mechanics 18, 33-58 (1986)

10. H.G. Hornung, M.I. Robinson, Journal of Fluid Mechanics 123, 155-164 (1982)

11. V.N. Uskov, M.V. Chernyshov, Journal of Applied Mechanics and Technical Physics 47, 492-504 (2006)

12. S. Mölder, Canadian Aeronautics and Space Institute Transactions 25, 44-49 (1979)

13. G. Ben-Dor, K. Takayama, AIAA Journal 24, 682-684 (1985)

14. M.V. Silnikov, M.V. Chernyshov, A.I. Mikhaylin, Acta Astronautica 109, 235-240 (2015) 